\title{
Pravastatin to prevent obstetrical complications in women with antiphospholipid syndrome
}

\author{
Maged M. Costantine \\ Department of Obstetrics and Gynecology, University of Texas Medical Branch, Galveston, Texas, USA.
}

\begin{abstract}
Pregnant women with antiphospholipid syndrome (APS) are at a high risk of obstetrical complications. The current standard of care, including the use of low-dose aspirin and heparin, has not been shown to prevent preeclampsia or intrauterine growth restriction (IUCR). Due to the similarities in pathophysiology among preeclampsia, IUGR, and atherosclerotic cardiovascular disease, statins have been proposed for treating and/or preventing these obstetrical complications. In this issue of the $J C I$, Lefkou et al. report on a small, observational trial that showed a dramatic improvement in both maternal and fetal/neonatal outcomes in women with APS given pravastatin after the onset of preeclampsia and/or IUGR compared with women in the control group. These results, along with other recent clinical studies, support further evaluation of statins for prevention of preeclampsia in a large-scale randomized clinical trial.
\end{abstract}

\section{Antiphospholipid syndrome and obstetrical morbidity}

The autoimmune, prothrombotic disorder antiphospholipid syndrome (APS) causes significant maternal and neonatal morbidity and mortality in pregnant women (1). APS is diagnosed based on characteristic clinical features, including vascular thrombosis, three or more pregnancy losses prior to the tenth week of gestation, one or more pregnancy losses after the tenth week of gestation, or premature delivery prior to 34 weeks gestation secondary to preeclampsia or intrauterine growth restriction (IUGR), and laboratory criteria that are confirmed 12 weeks apart (1). The pathogenesis of APS is complex, but involves exaggerated inflammation, endothelial dysfunction, and activation of the coagulation cascade, leading to thrombotic and obstetric morbidities (2). Moreover, poor placental implantation - which may be secondarily triggered by the above-mentioned mechanisms - early in pregnancy leads to reduced uteroplacental perfusion and angiogenic imbalance, together, which culminate clinically as ischemic placental disorder (IPD), including preterm preeclampsia and IUGR (3).

Low-dose aspirin and heparin have been suggested as first-line treatments for pregnant women with APS who have a history of recurrent pregnancy losses or arterial or venous thrombosis. Other therapies, such as corticosteroids or intravenous immunoglobulins, have not shown promise in preventing pregnancy losses (1). Most importantly, none of these therapies has been shown to prevent IPD (1). Any intervention that prevents the development of preeclampsia will have a substantial impact toward decreasing maternal mortality, especially in low-income countries, and will lessen the disease burden by reducing short- and long-term maternal (cardiovascular) and neonatal (prematurity related) complications (4-6).

Related Article: p. 2933

Conflict of interest: The author has declared that no conflict of interest exists.

Reference information: / Clin Invest. 2016;126(8):2792-2794. doi:10.1172/JCI89137.

\section{Rationale to use statins}

Preeclampsia and IUGR share several risk factors with adult atherosclerotic cardiovascular disease (CVD) $(6,7)$. Both inflammation and endothelial dysfunction are essential for the initiation and progression of these conditions $(6,8)$. In addition, women who develop IPD are at high risk of future CVD (5). Due to these similarities and the well-proven benefit of HMG-CoA reductase inhibitors (statins) for the prevention of CVD mortality and morbidities, there has been a growing interest in using statins to prevent IPD in high-risk pregnant women (6). The biological plausibility and the preclinical data from animal and placental models have been encouraging. In various preeclampsia animal models, pravastatin use has improved pregnancy outcomes, with reduced rates of pregnancy losses, restoration of the angiogenic balance, prevention of IUGR, and amelioration of the preeclampsia phenotype. These beneficial effects were demonstrated without any harmful effects on dams or any increase in the rates of anomalies in offspring (6, 9-12). The growing evidence from preclinical work that pravastatin improves pregnancy outcome, its biological plausibility, and the lack of agents effective at preventing placental disease led to several pilot clinical studies (13-15), including one by Lefkou et al. in this issue (16).

\section{Statins for treatment of preeclampsia and/or IUGR in women with APS: findings and limitations}

Lefkou et al. enrolled 21 women with APS and poor obstetrical history in a prospective cohort study. All patients received low-dose aspirin and low-molecular weight heparin (LMWH), as per standard local practice, and were monitored closely with serial ultrasound examinations to evaluate uterine and fetal vessels and closely assess fetal growth and with clinical evaluations to monitor for the development of preeclampsia and/or IUGR. 
After development of preeclampsia and/ or IUGR, ten patients were maintained on low-dose aspirin and LMWH (control cohort), while eleven patients were started on $20 \mathrm{mg}$ pravastatin/d along with low-dose aspirin and LMWH (pravastatin cohort). Compared with patients in the control cohort, those who received pravastatin had improved blood pressure and uterine artery blood flow, remained pregnant for a longer duration after initial diagnosis (13 weeks, interquartile range [IQR], 8-14 weeks vs. 4.5 weeks, IQR, 2-5), delivered at a later gestational age (GA) (36 weeks, IQR, 35-36 weeks vs. 26.5 weeks, IQR, 26-32), and had infants with higher birth weight at delivery (2,390 grams, IQR, 2,065-2,770 vs. 900 grams, IQR, 580-1,100). Pravastatin use was also associated with improved neonatal outcomes, as survival rate (to hospital discharge) was $100 \%$ in the pravastatin cohort compared with $45 \%$ in the control cohort. The rates of admission to the neonatal intensive care unit and later neurodevelopmental delays were reduced in the pravastatin cohort. The neonatal benefits in the pravastatin cohort were not associated with increased rates of maternal morbidities (16).

The work by Lefkou and colleagues is an important report that supports the potential utility of pravastatin to prevent pregnancy complications in high-risk pregnant women. However, findings from this study should be interpreted with caution and cannot be taken as conclusive due to several inherent limitations. Particularly, this was a small observational study that did not include a placebo arm. Patients were not randomly allocated to treatment or control groups, and there was no blinding. The control and treatment groups may not have been comparable. The choice of methyldopa as the agent of choice to control blood pressure was not well explained. Moreover, the sample size was very limited, and small studies tend to overestimate the effect size of an intervention. In this report, Lefkou et al. did not investigate the mechanisms that underlie the protective effects, but it is hoped that biological samples were collected for future investigation of angiogenic, inflammatory, endothelial, or other pleiotropic mechanisms. Moreover, while no increase in the rate of maternal mor- bidities was reported, the rates of statinassociated adverse events and liver and muscle enzyme concentrations were not included in the manuscript.

\section{Future directions}

The design of the study and timing of pravastatin administration (after the onset of preeclampsia and/or IUGR) is similar to that in the Statins to Ameliorate early onset Preeclampsia (StAmP) trial, a proofof-concept randomized trial that enrolled subjects in the United Kingdom (www.controlled-trials.com ISRCTN23410175) with an aim to evaluate whether pravastatin ameliorates the angiogenic imbalance in women with severe preeclampsia before 32 weeks' gestation. The results of the StAmP trial have not been reported yet. However, a recently completed pilot, multicenter, double-blind, placebo-controlled trial in the US randomized women at high risk for preeclampsia. Those with prior pregnancy that required delivery at less than 34 weeks' gestation for severe preeclampsia and were between $12^{0 / 7}$ and $16^{6 / 7}$ weeks' gestation were given daily pravastatin or placebo orally until delivery (www.clinicaltrials.gov NCT01717586) (15). Findings from the Eunice Kennedy Shriver National Institute of Child Health and Human Development - Obstetric Fetal Pharmacology Research Unit trial also support the beneficial role of pravastatin, as women who received pravastatin had lower rates of preeclampsia and indicated preterm delivery and better neonatal outcomes compared with those who received placebo, although the results of this trial were not as dramatic as the results of Lefkou et al. (16). Importantly, the US trial demonstrated an improved angiogenic profile with the use of pravastatin and no difference in the rate of adverse events (based on clinical and laboratory observations) or congenital anomalies. Although pravastatin reduced maternal cholesterol concentrations, umbilical cord cholesterol concentrations and infant birth weight were not different between the treatment and control groups (15).

Pravastatin is one of the original statins that was shown to be effective in preventing cardiovascular mortality and morbidity. It has unique pharmacokinetic and physiochemical properties and is one of the most hepatoselective and hydrophilic (polar) statins (17). Pravastatin's inhibition of
HMG-CoA reductase is limited compared with that of other statins and relatively minimal in nonhepatocyte cells. Pravastatin is cleared through renal and hepatic routes, which reduces the need for dose reduction in the case of kidney or liver impairment. Moreover, it is not dependent on CYP3A metabolism; therefore, there are no clinically relevant pharmacokinetic interactions between pravastatin and CYP3A inhibitors. Additionally, pravastatin is a substrate of placental efflux transporters such as P-glycoprotein and multidrug resistance-associated protein $2(17,18)$. Consistent with these characteristics, recent transplacental studies have shown that the drug's ability to cross the placenta was limited and clearance was higher in the fetal-to-maternal direction than the maternal-to-fetal direction (18). The US randomized controlled trial additionally showed that renal clearance of pravastatin is higher in pregnancy compared with in the nonpregnant state, adding uncertainty to the ideal dose with the safest profile to be used in high-risk pregnant women (15). Data from pregnancy exposure cohorts do not support the teratogenicity claims of pravastatin $(6,19)$.

In conclusion, several small pilot studies, including the one by Lefkou and colleagues, support the biologic plausibility and are concordant with the preclinical work that shows pravastatin is able to prevent preeclampsia in various groups of high-risk pregnant women. We have come a long way from the time when giving statins to pregnant women was considered taboo. However, before statin administration becomes part of routine clinical practice, a large, well-designed, and adequately powered randomized controlled trial is needed. Until then, pravastatin use in pregnant women remains investigational.

\section{Acknowledgments}

This work is supported in part by a grant from The Eunice Kennedy Shriver National Institute of Child Health and Human Development (NICHD) (2U54HD047891-11). The manuscript does not necessarily represent the official views of the NICHD or the NIH.

Address correspondence to: Maged Costantine, 301 University Blvd., Galveston, Texas 77555-0587, USA. Phone: 409.772.1571; E-mail:mmcostan@utmb.edu. 
1. American College of Obstetricians Gynecologists. Antiphospholipid Syndrome. Practice bulletin No. 132. Obstet Gynecol. 2012;120(6):1514-1521.

2. Giannakopoulos B, Krilis SA. The pathogenesis of the antiphospholipid syndrome. $N$ Engl J Med 2013;368(11):1033-1044

3. Ananth CV. Ischemic placental disease: a unifying concept for preeclampsia, intrauterine growth restriction, and placental abruption. Semin Perinatol. 2014;38(3):131-132.

4. Mongraw-Chaffin ML, Cirillo PM, Cohn BA. Preeclampsia and cardiovascular disease death: prospective evidence from the child health and development studies cohort. Hypertension. 2010;56(1):166-171.

5. McDonald SD, Malinowski A, Zhou Q, Yusuf S, Devereaux PJ. Cardiovascular sequelae of preeclampsia/eclampsia: a systematic review and meta-analyses. Am Heart J. 2008;156(5):918-930.

6. Costantine MM, Cleary K, for the Eunice Kennedy Shriver National Institute of Child Health Human Development Obstetric-Fetal Pharmacology Research Units Network. Pravastatin for the prevention of preeclampsia in high risk pregnant women. Obstet Gynecol. 2013;121(2 pt 1):349-353.
7. Berends AL, et al. Shared constitutional risks for maternal vascular-related pregnancy complications and future cardiovascular disease. Hypertension. 2008;51(4):1034-1041.

8. Hansson GK. Inflammation, atherosclerosis, and coronary artery disease. $N$ Engl J Med. 2005;352(16):1685-1695.

9. Costantine MM, et al. Using pravastatin to improve the vascular reactivity in a mouse model of soluble fms-like tyrosine kinase-1induced preeclampsia. Obstet Gynecol. 2010;116(1):114-120.

10. Ahmed A, Singh J, Khan Y, Seshan SV, Girardi G. A new mouse model to explore therapies for preeclampsia. PLoS One. 2010;5(10):e13663.

11. Kumasawa K, et al. Pravastatin induces placental growth factor (PGF) and ameliorates preeclampsia in a mouse model. Proc Natl Acad Sci U S A. 2011;108(4):1451-1455.

12. Saad AF, et al. Effects of pravastatin on angiogenic and placental hypoxic imbalance in a mouse model of preeclampsia. Reprod Sci. 2014;21(1):138-145.

13. Brownfoot FC, et al. Effects of pravastatin on human placenta, endothelium, and women with severe preeclampsia. Hypertension. 2015;66(3):687-697.

14. Chaiworapongsa T, et al. Pravastatin to prevent recurrent fetal death in massive perivillous fibrin deposition of the placenta (MPFD). JMatern Fetal Neonatal Med. 2016;29(6):855-862.

15. Costantine MM, et al. Safety and pharmacokinetics of pravastatin used for the prevention of preeclampsia in high-risk pregnant women: a pilot randomized controlled trial. Am J Obstet Gynecol. 2016;214(6):720.e1-720.e17.

16. Leftkou E, Mamopoulos A, Dagklis T, Vosnakis C, Rousso D, Girardi G. Pravastatin improves pregnancy outcomes in obstetric antiphospholipid syndrome refractory to antithrombotic therapy. J Clin Invest. 2016;126(8):2933-2940.

17. Hatanaka T. Clinical pharmacokinetics of pravastatin: mechanisms of pharmacokinetic events. Clin Pharmacokinet. 2000;39(6):397-412.

18. Nanovskaya TN, Patrikeeva SL, Paul J, Costantine MM, Hankins GD, Ahmed MS. Transplacental transfer and distribution of pravastatin. Am JObstet Gynecol. 2013;209(4):373.e1-373.e5.

19. Bateman BT, et al. Statins and congenital malformations: cohort study. BMJ. 2015;350:h1035. 